Available online at www.sciencedirect.com

\title{
Conformational Flexibility of Four-way Junctions in RNA
}

\author{
Sungchul Hohng ${ }^{1}$, Timothy J. Wilson ${ }^{2}$, Elliot Tan ${ }^{1}$, Robert M. Clegg ${ }^{1}$ \\ David M. J. Lilley ${ }^{2 *}$ and Taekjip Ha ${ }^{1 *}$
}

${ }^{1}$ Department of Physics University of Illinois at Urbana-Champaign, Urbana IL 61801, USA

${ }^{2}$ Cancer Research UK Nucleic Acid Structure Research Group Department of Biochemistry The University of Dundee Dundee DD1 5EH, UK

\begin{abstract}
Helical junctions are common architectural features in RNA. They are particularly important in autonomously folding molecules, as exemplified by the hairpin ribozyme. We have used single-molecule fluorescence spectroscopy to study the dynamic properties of the perfect $(4 \mathrm{H})$ four-way helical junction derived from the hairpin ribozyme. In the presence of $\mathrm{Mg}^{2+}$, the junction samples parallel and antiparallel conformations and both stacking conformers, with a bias towards one antiparallel stacking conformer. There is continual interconversion between the forms, such that there are several transitions per second under physiological conditions. Our data suggest that interconversion proceeds via an open intermediate with reduced cation binding in which coaxial stacking between helices is disrupted. The rate of interconversion becomes slower at higher $\mathrm{Mg}^{2+}$ concentrations, yet the activation barrier decreases under these conditions, indicating that entropic effects are important. Transitions also occur in the presence of $\mathrm{Na}^{+}$only; however, the coaxial stacking appears incomplete under these conditions. The polymorphic and dynamic character of the four-way RNA junction provides a source of structural diversity, from which particular conformations required for biological function might be stabilised by additional RNA interactions or protein binding.
\end{abstract}

(C) 2004 Published by Elsevier Ltd.

Keywords: RNA structures; single molecule spectroscopy; fluorescence resonance energy transfer; hairpin ribozyme

\section{Introduction}

Helical junctions are very common in natural RNA species. For example, 18 three-way and fourway junctions are present in the structure of $23 \mathrm{~S}$ rRNA within the $50 \mathrm{~S}$ ribosomal subunit. Junctions are frequently found in small, autonomously folding RNA species, where they exert a powerful influence on the folding of the RNA. This is exemplified by the nucleolytic ribozymes, where the hairpin ribozyme contains an important fourway junction, ${ }^{1-5}$ and the hammerhead ${ }^{6,7}$ and VS ribozymes ${ }^{8}$ have one and two three-way junctions, respectively.

Helical junctions in nucleic acids are classed according to the number of helical arms, and by

Abbreviation used: FRET, fluorescence resonance energy transfer.

E-mail addresses of the corresponding authors: d.m.j.lilley@dundee.ac.uk; tjha@uiuc.edu the presence or absence of additional unpaired nucleotides present at the branchpoint. ${ }^{9}$ The simplest four-way helical junction (a $4 \mathrm{H}$ junction ${ }^{9}$ ) has fully base-paired helices with no additional unpaired bases; in DNA this is often referred to as a Holliday junction. ${ }^{10}$ Four-way junctions adopt a compact fold by pairwise coaxial stacking of helices. ${ }^{11}$ While this requires the binding of metal ions for DNA junctions, ${ }^{12,13}$ no stable unstacked conformation has been detected for any $4 \mathrm{H}$ RNA junction studied. ${ }^{14}$ Formation of a stacked structure lowers the symmetry of the junction from 4-fold to 2-fold, and thus creates two distinguishable kinds of strand. Continuous strands turn around a single axis running through the coaxially stacked helices, while exchanging strands cross between the two coaxial pairs at the point of strand exchange.

In principle, $4 \mathrm{H}$ junctions can adopt a number of different conformations depending upon local base sequence and conditions, summarised in Figure 1. (1) There are two ways in which the arms can be 


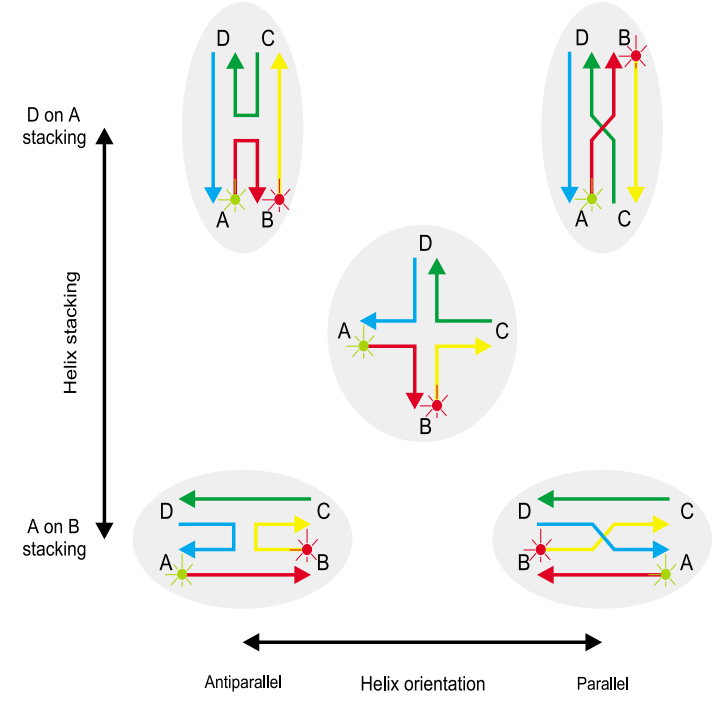

Figure 1. The possible conformations of a four-way helical junction. The junction has four arms, named A through D. This is shown in open form (centre). The strands are shown differentiated by colour, with polarities indicated by the arrowheads. The junction folds by coaxial stacking of helical arms in pairs, resulting in 2-fold symmetrical structures. This divides the component strands into two classes. Continuous strands have a single axis, while exchanging strands pass between axes at the crossover at the centre of the junction. The junction can adopt a parallel structure (right-hand side) where the continuous strands proceed in the same direction, or antiparallel (left-hand side), where they run in opposite directions. For each of these structures there are two possible stacking conformers. Stacking of helices D on A plus C on B generates the upper structures, while $A$ on $B$ plus $D$ on $C$ results in the lower structures. Note that the transition between stacking conformers changes the nature of each strand, so that continuous strands become exchanging strands and vice versa. If the axes are not exactly side-by-side, then right and left-handed forms of each structure are also possible. In the FRET studies, donor and acceptor fluorophores are attached to the $5^{\prime}$ termini of specific strands, as illustrated for the $\mathrm{AB}$ vector. $\mathrm{Cy} 3$ donor (green disk) is attached to the A arm, and Cy5 acceptor (red disk) to the $\mathrm{B}$ arm. Note that the two fluorophores are only close in the antiparallel D on A stacking conformer (top left).

paired for stacking. Arm A could be stacked coaxially upon arm B (and thus arm D on C), or alternatively arm D could stack with arm A (and hence $\mathrm{C}$ with $\mathrm{B}$ ). These are distinct conformers of the junctions, which we refer to as stacking conformers. (2) We can distinguish between different relative orientations of the two stacked helical pairs. These are depicted in their extreme forms in Figure 1. In the antiparallel forms, the polarity of the continuous strands run in opposite directions, while in the parallel forms they have the same direction. This property can be considered to be a rotation of one stacked helical pair relative to the other, and could take any angle between the parallel and antiparallel forms. Generally the axes would subtend some intermediate angle, which would be closer to one form or the other, but not necessarily side-by-side. If the angle is exactly $90^{\circ}$, then the structure is neither parallel nor antiparallel. (3) If the helices are not side-by-side, then there is a third property that must be considered, that of handedness. Since the two axes are displaced laterally by the width of a helix, both right and left-handed forms of any X-shaped conformation are theoretically possible.

Thus in total we can envision eight different possible conformational forms of a $4 \mathrm{H}$ junction. A DNA $4 \mathrm{H}$ junction has a strong propensity to adopt a right-handed, antiparallel conformation. ${ }^{12,13}$ However, two alternative stacking conformers are still possible, which can be more or less biased towards one form depending on the sequence. We have recently shown that there is continual exchange between stacking conformers in DNA junctions. ${ }^{15}$

Much less is currently known about the conformational possibilities and propensities in RNA $4 \mathrm{H}$ junctions, yet this information is important if we are to understand the contribution of these motifs to the structure and folding of RNA containing them. A good example is the $4 \mathrm{H}$ junction that is an integral part of the hairpin ribozyme. ${ }^{1-5}$ The active site of the ribozyme is created by the intimate interaction between two internal loops, that are present on two adjacent arms of the fourway junction. Although the ribozyme is active in the absence of the junction, ${ }^{16}$ its presence accelerates the ion-induced folding of the ribozyme by 500 -fold, ${ }^{5}$ and reduces the required ionic concentration by three orders of magnitude and thus allows folding to proceed under physiological conditions. ${ }^{1,2,4}$ We have therefore sought to understand the conformational properties of this junction in the absence of the loops, i.e. as a simple $4 \mathrm{H}$ junction. We have previously used fluorescence resonance energy transfer (FRET) between donoracceptor fluorophore pairs attached to the ends of different helices as a means of analysing the conformation and transitions of junctions. ${ }^{17}$ However, because potential interconversion processes between the conformations illustrated in Figure 1 cannot be synchronised, we have used singlemolecule FRET spectroscopy ${ }^{18}$ to analyse the presence of the different conformers, and their rates of interconversion. This technique has proved to be very useful in revealing detailed kinetic information for RNA folding and catalysis. ${ }^{5,19-21}$ Single-molecule FRET analysis of the RNA fourway junction has revealed a rich conformational landscape populated by parallel and antiparallel forms of the junction, and both stacking conformers. These undergo continual dynamic interconversion processes. RNA four-way junctions are therefore structurally highly polymorphic, which must be taken into account in considering the folding properties of large RNA species. 


\section{Results}

\section{Analysis of conformational forms in a four-way RNA junction}

We have studied the simple $4 \mathrm{H}$ junction derived from the hairpin ribozyme, with four perfectly base-paired arms. The arms are named A through $\mathrm{D}$ sequentially around the junction, in the conventional manner (Figure 1). ${ }^{1}$ We have analysed FRET between donor (Cy3) and acceptor (Cy5) fluorophores terminally attached to different helical arms of the junction in order to distinguish the various possible conformations of the junction shown schematically in Figure 1. Fluorescent vectors are named according to the arms carrying the donor and acceptor in that order; for example the vector $\mathrm{DB}$ carries $\mathrm{Cy} 3$ on the $5^{\prime}$ end of the $\mathrm{D}$ arm, and Cy5 on the $5^{\prime}$ end of the $\mathrm{B}$ arm. We have used three such vectors in this study, AB, DB and AD. These were chosen to report on the various possible conformations of the junction discussed above.

\section{$A B$ vector, antiparallel $D$ on $A$ conformer}

This is illustrated in Figure 1. It can be seen that the donor-acceptor pair should only be physically close (and therefore result in elevated energy transfer between the fluorophores) for the antiparallel conformer of the D on A stacked form. Either rotation to the parallel form, or conversion to the alternative (A on B) stacking conformer leads to separation of the fluorophores, and thus lowered FRET efficiency.

\section{$A D$ vector, antiparallel $A$ on $B$ conformer}

The fluorophores will only be close in the antiparallel form of the alternative stacking conformer with A on B stacking.

\section{DB vector, parallel conformers}

In this vector the fluorophores will be close in either of the parallel forms. It will thus distinguish parallel conformers (as a group), but not which stacking conformer is present.

The four conformers that are detected by the high-FRET efficiency forms of the three vectors should form a complete set of pairwise, coaxially stacked species. Note that this approach does not distinguish between right and left-handed forms of these conformers. The following experiments have been performed using single-molecule FRET approaches, with the junctions immobilised onto a glass surface via biotin covalently attached to the $5^{\prime}$ terminus of the $C$ arm in each case.

\section{Presence and interconversion of multiple conformations of the RNA junction}

We have studied the conformation of the hairpin

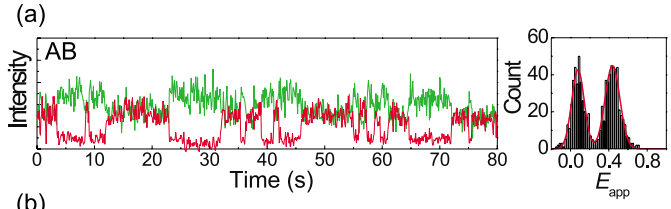

(b)
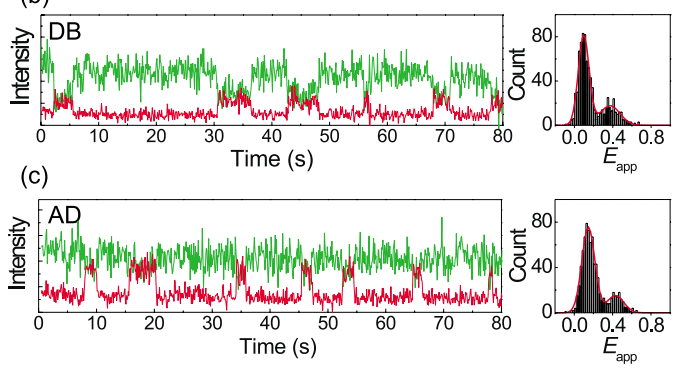

Figure 2. Stacking conformer and parallel-antiparallel transitions in the four-way RNA junction. Singlemolecule time records ( $100 \mathrm{~ms}$ resolution) for the three vectors of the hairpin junction recorded in the presence of $10 \mathrm{mM}$ Hepes (pH 7.5), $20 \mathrm{mM} \mathrm{MgCl}, 50 \mathrm{mM} \mathrm{NaCl}$ at $9{ }^{\circ} \mathrm{C}$. Corresponding FRET histograms are shown on the right. The data have been fitted to two Gaussian distributions (red lines). (a) A typical time trace for the $A B$ vector (left), showing clear anti-correlation between fluorescent emission arising from the donor (green) and acceptor (red). The FRET histogram (right) shows that the molecule spends approximately equal amounts of time in the high and low-FRET states. (b) and (c) Time traces and FRET histograms for the $\mathrm{DB}$ vector and AD vector, respectively. A significant bias to a low-FRET state is seen for both vectors.

junction by single-molecule FRET analysis using the three vectors $\mathrm{AB}, \mathrm{DB}$ and $\mathrm{AD}$. Experiments were performed in the presence of $20 \mathrm{mM} \mathrm{MgCl}$, $50 \mathrm{mM} \mathrm{NaCl}$ at $9{ }^{\circ} \mathrm{C}$. Representative time traces for the three vectors are shown in Figure 2. All three exhibit two-state fluctuations in FRET efficiency $\left(E_{\mathrm{app}}\right.$ ) between $\sim 0.4$ and $\sim 0.1$. In each case, the higher value corresponds to a conformation that places the two fluorophores relatively close together in space. We therefore conclude that the junction has significant populations of antiparallel D-on-A conformer (high FRET AB vector), antiparallel A-on-B conformer (high FRET AD vector), and either or both parallel conformers (high FRET DB vector).

The transitions between high and low FRET states indicate the existence of a dynamic equilibrium between these alternative conformations of the junction. Figure 3 shows histograms of the dwell times $\left(t_{\text {High }}\right.$ and $\left.t_{\text {Low }}\right)$ for the high and low FRET states of the three vectors. Single exponential decay fits yield the forward $\left(k_{\mathrm{f}}=1 / t_{\text {Low }}\right.$ : low to high FRET) and backward $\left(k_{\mathrm{b}}=1 / t_{\text {High }}\right.$ : high to low FRET) rate constants (Table 1). From these rate constants, we can calculate the probabilities with which the junction remains in the state with high FRET for each vector, $P_{\text {High }}=k_{\mathrm{f}} /\left(k_{\mathrm{f}}+k_{\mathrm{b}}\right)$. If the junction adopts a pairwise coaxially stacked structure (illustrated in Figure 1), the high FRET states of the three vectors should be mutually 

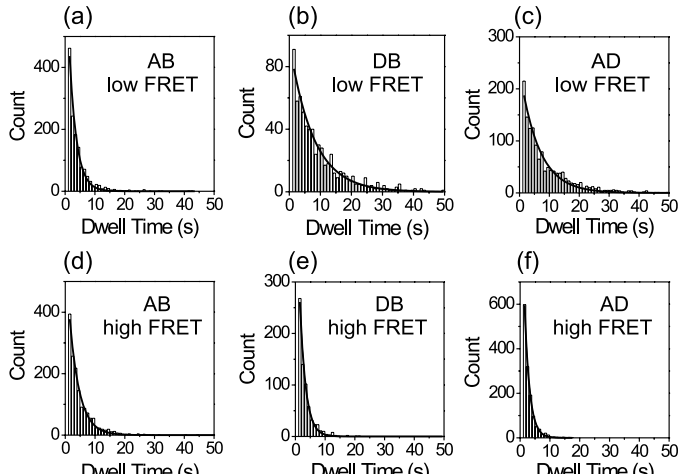

$$
\text { (f) }
$$

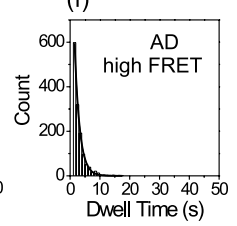

Figure 3. Dwell time histograms of the low and highFRET states of the three vectors of the hairpin junction. Data were recorded in the presence of $10 \mathrm{mM}$ Hepes (pH 7.5), $20 \mathrm{mM} \mathrm{MgCl}_{2}, 50 \mathrm{mM} \mathrm{NaCl}$ at $9{ }^{\circ} \mathrm{C}$. Dwell times from more than 200 single molecules were accumulated with a bin size of $1 \mathrm{~s}$. Each histogram has been fitted with a single exponential function (continuous lines). Decay rates are summarised in Table 1. (a) -(c) Dwell time histograms of the high-FRET states of the $A B, D B$, and $A D$ vectors, respectively. (d)-(f) Dwell time histograms of the low-FRET states of the $\mathrm{AB}, \mathrm{DB}$ and $\mathrm{AD}$ vectors, respectively.

exclusive and form a complete set. Therefore, the sum of the three probabilities $\left(P_{\text {total }}=P_{\text {High, AB }}+\right.$ $\left.P_{\text {High,AD }}+P_{\text {High,DB }}\right)$ should be $100 \%$. The result is $99 \%$ based on the rate constants determined by fitting the dwell time histograms. We can also determine $P_{\text {High }}$ values by adding all dwell times together and taking the ratio, $P_{\text {High }}=$ $\sum t_{\mathrm{High}} /\left(\sum t_{\text {High }}+\sum t_{\text {Low }}\right)$, and $P_{\text {total }}$ thus obtained is $96 \%$. This is therefore consistent with a model in which the helices are essentially fully stacked in pairs under the conditions of the experiment. This result is also consistent with previous studies that showed stacking was preserved at all $\mathrm{Mg}^{2+}$ concentrations, with a dominant antiparallel D-on-A stacked structure in the presence of $20 \mathrm{mM}$ $\mathrm{Mg}^{2+} ., 22$

\section{Dependence of the rates of conformer transition on magnesium ion concentration}

Rates $k_{\mathrm{f}}$ and $k_{\mathrm{b}}$ have been measured for all three vectors for $\mathrm{Mg}^{2+}$ concentrations over the range from $1 \mathrm{mM}$ to $100 \mathrm{mM}$ (Figure 4). While the ratios of the two rate constants do not change significantly as a function of $\mathrm{Mg}^{2+}$ concentration, the

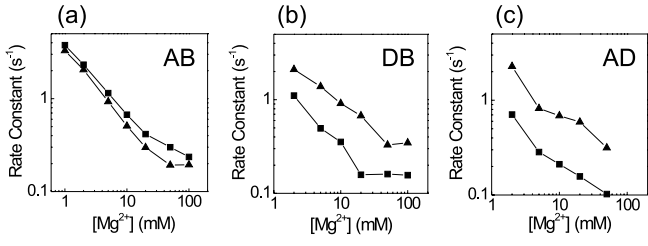

Figure 4. Forward and backward rate constants as a function of $\mathrm{Mg}^{2+}$ concentration for the three vectors. Forward rate constants (squares) and backward rate constants (triangles) of the $\mathrm{AB}(\mathrm{a}), \mathrm{DB}(\mathrm{b})$ and $\mathrm{AD}(\mathrm{c})$ vectors in the presence of $10 \mathrm{mM}$ Hepes ( $\mathrm{pH}$ 7.5), $50 \mathrm{mM} \mathrm{NaCl}$ at $9^{\circ} \mathrm{C}$.

rates themselves change by a factor of more than 20 , becoming slower at higher $\mathrm{Mg}^{2+}$ concentrations as we have reported previously for the $\mathrm{AB}$ vector. ${ }^{5}$

Ensemble FRET measurements have previously shown that a significant bias toward the antiparallel D-on-A stacked form develops at elevated $\mathrm{Mg}^{2+}$ concentrations. ${ }^{17}$ Ensemble data obtained for the $A B$ vector also show an increase in FRET at higher $\mathrm{Mg}^{2+}$ concentrations (Figure 5(a)). This could arise from two independent sources. (1) The FRET values of each state could change. (2) The high FRET state could become more favoured at higher $\mathrm{Mg}^{2+}$ concentration. Single-molecule FRET histograms show that the FRET efficiency of the high FRET state increases with increasing $\mathrm{Mg}^{2+}$ concentrations (Figure 5(b) and (c)). This is probably due to a change in the inter-helical angles of the junction that brings the $\mathrm{A}$ and $\mathrm{B}$ helices closer together. In principle, other effects may cause the apparent increase in FRET, such as the fluorophore properties being affected by $\mathrm{Mg}^{2+}$. However, this is unlikely because the AD vector did not show an increase in the FRET values of the high FRET states with $\mathrm{Mg}^{2+}$ (Figure 5(c)). In agreement with previous single-molecule studies, ${ }^{5}$ we also observed a small increase in $P_{\text {High AB }}$ with increasing $\mathrm{Mg}^{2+}$ concentration (Figure 5(d)). Overall, both of these effects, conformer bias change and interhelical angle change, combine to produce the ensemble FRET increase of the $A B$ vector with increasing $\mathrm{Mg}^{2+}$ concentration.

\section{Temperature dependence of transition rates}

In order to understand the nature of the free energy barriers for the conformational transitions, we performed temperature dependence studies of

Table 1. Transition rate constants for the three vectors of the hairpin junction in the presence of $10 \mathrm{mM}$ Hepes (pH 7.5), $20 \mathrm{mM} \mathrm{MgCl} 2,50 \mathrm{mM} \mathrm{NaCl}$ at $9{ }^{\circ} \mathrm{C}$

\begin{tabular}{llcccr}
\hline & $k_{\mathrm{f}}\left(\mathrm{s}^{-1}\right)$ & $k_{b}\left(\mathrm{~s}^{-1}\right)$ & $P_{\text {dwell time }}(\%)$ & $P_{\text {rate constants }}(\%)$ & \multicolumn{2}{c}{$\Delta H^{*}\left(\mathrm{kcal} \mathrm{mol}^{-1}\right)$} \\
\hline AB & $0.42 \pm 0.01$ & $0.30 \pm 0.01$ & 55 & 59 & $22.3 \pm 1.1$ \\
DB & $0.16 \pm 0.01$ & $0.67 \pm 0.02$ & 19 & 21 & $20.5 \pm 2.6$ \\
AD & $0.16 \pm 0.01$ & $0.59 \pm 0.01$ & Sum $=96$ & Sum $=99$ & $23.4 \pm 0.4$
\end{tabular}

The rate constants were calculated by fitting the dwell time histograms (as shown in Figure 3) with single-exponential functions. The probabilities of a vector being in the high-FRET state were obtained in two ways, as described in the text. Activation enthalpies were measured from the temperature dependence of transition rates, as shown in Figure 6. 
(a)
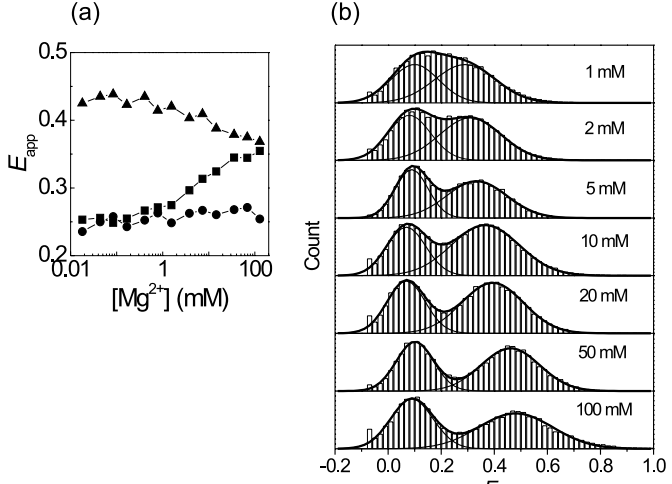

(d)
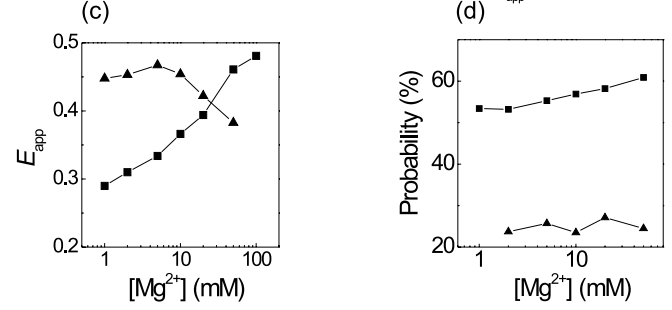

Figure 5. Distribution of FRET efficiencies $\left(E_{\mathrm{app}}\right)$ as a function of $\mathrm{Mg}^{2+}$ concentration. Experiments were performed in the presence of $10 \mathrm{mM}$ Hepes ( $\mathrm{pH}$ 7.5), $50 \mathrm{mM} \mathrm{NaCl}$ plus the indicated $\mathrm{MgCl}_{2}$ concentration at $9{ }^{\circ} \mathrm{C}$. (a) Ensemble FRET efficiencies as a function of $\mathrm{Mg}^{2+}$ concentration for $\mathrm{AB}$ (squares), $\mathrm{AD}$ (triangles), and DB (circles) vectors. (b) FRET histograms for the AB vector at the indicated $\mathrm{Mg}^{2+}$ concentration, and their two-Gaussian fits. (c) Mean FRET efficiency for the high FRET state for $\mathrm{AB}$ (squares) and $\mathrm{AD}$ (triangles) vectors. (d) Probabilities of high FRET states for the AB (squares) and $\mathrm{AD}$ (triangles) vectors as a function of $\mathrm{Mg}^{2+}$ concentration.

the transition rates for all three vectors in $20 \mathrm{mM}$ $\mathrm{MgCl}_{2}, 50 \mathrm{mM} \mathrm{NaCl}$. Figure 6(a)-(c) shows the transition rate on a logarithmic scale as a function of inverse temperature (Arrhenius plot). The equilibrium between states does not change appreciably within the temperature range studied (data not shown) and the transition rates plotted are the sum of $k_{\mathrm{f}}$ and $k_{\mathrm{b}}$ obtained from autocorrelation analysis because transitions between states are too fast for reliable dwell time analysis at the highest temperatures used. Autocorrelation analysis and dwell time analysis gave identical results at lower temperatures. The data shown in the Arrhenius plots are linear within our precision of measurements with the correlation coefficients of 0.99 for all cases, suggesting that the enthalpic and entropic barriers are not significantly dependent on the temperature within the temperature range studied. Therefore, the data have been fitted to the Arrhenius equation $\left(k_{\mathrm{f}}+\right.$ $\left.k_{\mathrm{b}}\right)=A \exp \left(-\Delta H^{\ddagger} / R T\right)$, from which the activation enthalpies $\left(\Delta H^{\ddagger}\right)$ have been obtained (Table 1). Interestingly, the activation barriers are closely similar for all three vectors, with $\Delta H^{\ddagger} \sim 22 \mathrm{kcal} \mathrm{mol}^{-1}$, suggesting that mechanistically similar rate-limiting steps are involved for the interconversions observed using the three vectors.
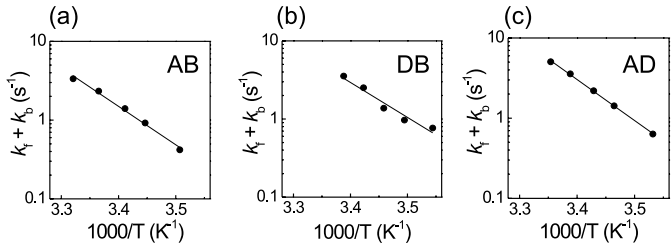

(d)

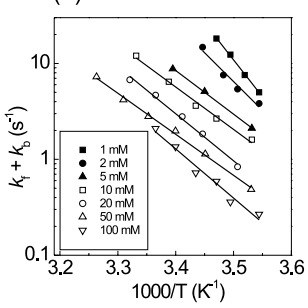

(e)

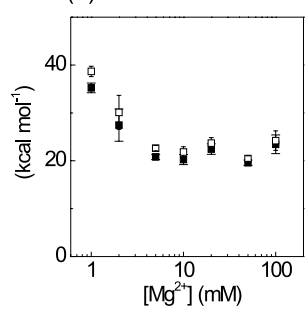

Figure 6. Temperature dependence of transition rates for the hairpin junction. The sum of forward and backwards rates of transition $\left(k_{\mathrm{f}}+k_{\mathrm{b}}\right)$ in the presence of $10 \mathrm{mM}$ Hepes (pH 7.5), $20 \mathrm{mM} \mathrm{MgCl}_{2}, 50 \mathrm{mM} \mathrm{NaCl}$ were measured by autocorrelation analysis. (a) - (c) The data are presented as Arrhenius plots of $\log \left(k_{\mathrm{f}}+k_{\mathrm{b}}\right)$ as a function of inverse absolute temperature, showing the data (filled circles) and fit to the Arrhenius equation (line) for the AB (a), DB (b) and AD (c) vectors. The activation energies are listed in Table 1. (d) Transition rates $\left(k_{\mathrm{f}}+k_{\mathrm{b}}\right)$ for the $\mathrm{AB}$ vector as a function of $\mathrm{Mg}^{2+}$ concentration. (e) Plot of activation energies (filled squares) and pre-exponential factors (open squares) for the $A B$ vector as a function of $\mathrm{Mg}^{2+}$ concentration.

\section{Dependence of activation energies on $\mathrm{Mg}^{2+}$ concentration}

Figure 6(d) shows Arrhenius plots of the transition rates $\left(k_{\mathrm{f}}+k_{\mathrm{b}}\right)$ of the $\mathrm{AB}$ vector at various $\mathrm{Mg}^{2+}$ concentrations, fitted to the Arrhenius equation. The resulting values of $\Delta H^{\ddagger}$ and $R T \ln A$ are shown in Figure 6(e) as a function of $\mathrm{Mg}^{2+}$ concentration. We observe no decrease of $\Delta H^{\ddagger}$ with decreasing $\mathrm{Mg}^{2+}$ concentration, despite the substantial increase in transition rates. On the contrary, $\Delta H^{\ddagger}$ increases significantly with decreasing $\mathrm{Mg}^{2+}$ concentrations below $10 \mathrm{mM}$. $\ln A$, which contains the entropic gain $\Delta S^{\ddagger}$, also increases with decreasing $\mathrm{Mg}^{2+}$ concentration. The conformational transition is entropically favoured at low $\mathrm{Mg}^{2+}$ concentration, showing a compensation between changes in entropy and enthalpy (Figure 6(e)).

\section{Transitions occurring in high $\mathrm{Na}^{+}$ concentration in the absence of $\mathrm{Mg}^{2+}$}

Monovalent ions are generally less effective in folding RNA molecules. However, if the role of cations in RNA folding is simply the screening of the negative charges of phosphate groups, monovalent cations are expected to induce folding at high concentrations. Figure 7 shows typical time traces for the three vectors in the presence of $1 \mathrm{M}$ $\mathrm{NaCl}$ and in the absence of $\mathrm{Mg}^{2+}$. As expected, $4 \mathrm{H}$ junctions display clearly resolvable conformational 

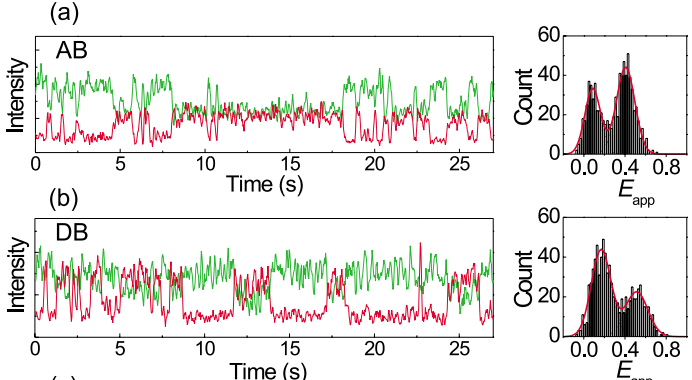

(c)
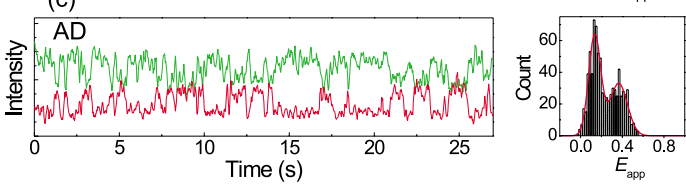

Figure 7. Stacking conformer and parallel-antiparallel transitions in the four-way RNA junction in the presence of a high concentration of $\mathrm{Na}^{+}$and no $\mathrm{Mg}^{2+}$. Singlemolecule time records (33 ms time resolution) for the three vectors of the hairpin junction recorded in the presence of $10 \mathrm{mM}$ Hepes $(\mathrm{pH} 7.5), 1 \mathrm{M} \mathrm{NaCl}$. The $\mathrm{AB}$ vector was studied at $10^{\circ} \mathrm{C}$, while the $\mathrm{DB}$ and $\mathrm{AD}$ vectors were recorded at $8{ }^{\circ} \mathrm{C}$. Corresponding FRET histograms are shown on the right. The data have been fitted to two Gaussian distributions (red lines). (a) Time trace for the $A B$ vector (left), showing fluorescent emission arising from the donor (green) and acceptor (red); only a portion of the trace is shown in order to reveal the fluctuations. The FRET histogram (right) shows the proportion of time that the molecules spend in the high- and low-FRET states. (b) and (c) Time traces and FRET histograms for the $\mathrm{DB}$ and $\mathrm{AD}$ vector, respectively.

fluctuations under these conditions. Compared to the data in $20 \mathrm{mM} \mathrm{Mg}^{2+}$, the bias to the high FRET states increases for all vectors. The net result is that the sum of probabilities of the high FRET states for all three vectors well exceeds 100\% calculated both by dwell time analysis and measurement of rate constants (145\% and 150\%, respectively; Table 2). This indicates that the high FRET states of the three vectors are not mutually exclusive and the model shown in Figure 1 does not fully describe the behaviour of the junction. Thus stacking is not complete in the presence of $\mathrm{Na}^{+}$alone, whereby the unstacked form could spend a portion of the time in a conformation that places the fluorophores in proximity, creating an apparent bias towards the high FRET state.

\section{Dependence of transition rates on $\mathrm{Na}^{+}$ concentration in the presence of $\mathrm{Mg}^{2+}$}

$\mathrm{Na}^{+}$alone can stabilise various conformations, as seen from the slower fluctuations at higher $\mathrm{Na}^{+}$ concentrations. To explore the effect of monovalent ions in the presence of $\mathrm{Mg}^{2+}$, we studied how the kinetics of the $\mathrm{AB}$ vector depend on $\mathrm{Na}^{+}$concentration at various $\mathrm{Mg}^{2+}$ concentrations (Figure 8). In the absence of $\mathrm{Mg}^{2+}$, the dependence of transition rates as a function of $\mathrm{Na}^{+}$is similar to that for $\mathrm{Mg}^{2+}$; at high cation concentration, transition

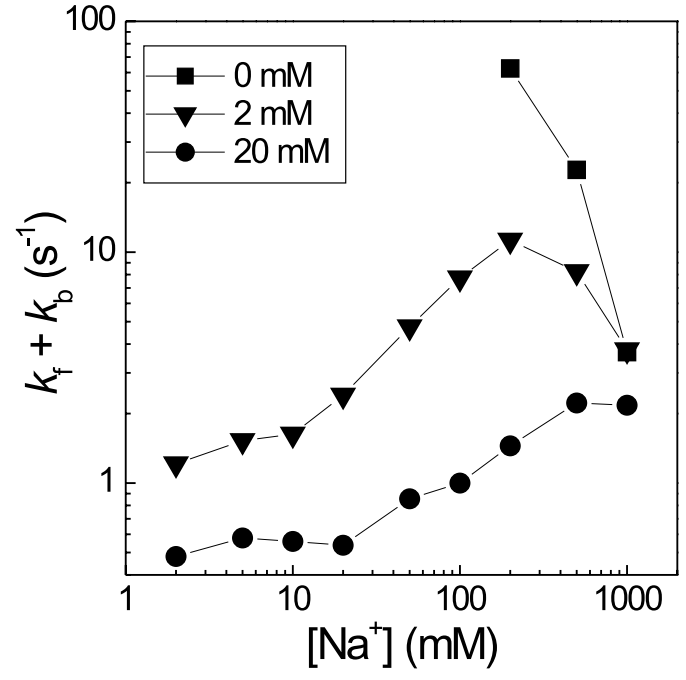

Figure 8. Transition rates as a function of $\mathrm{Na}^{+}$concentration at fixed concentrations of $\mathrm{Mg}^{2+}$. Plot of transition rates $\left(k_{\mathrm{f}}+k_{\mathrm{b}}\right)$ measured by correlation analysis for the $\mathrm{AB}$ vector of the hairpin junction at $11{ }^{\circ} \mathrm{C}$ as a function of $\mathrm{Na}^{+}$concentration in a background concentration of $\mathrm{Mg}^{2+}$ of $0 \mathrm{mM}$ (squares), $2 \mathrm{mM}$ (triangles), or $20 \mathrm{mM}$ (circles).

rates become slower. However, the actual rates are significantly higher in the presence of $\mathrm{Na}^{+}$, compared to those in $\mathrm{Mg}^{2+}$. In the presence of $2 \mathrm{mM}$ $\mathrm{Mg}^{2+}, \mathrm{Na}^{+}$at low concentrations increases the transition rates, but this effect is reversed above $200 \mathrm{mM} \mathrm{Na}^{+}$. A similar behaviour occurs in the presence of $20 \mathrm{mM} \mathrm{Mg}^{2+}$, but the turning point is shifted to higher $\mathrm{Na}^{+}$concentration. It appears that $\mathrm{Na}^{+}$induces the RNA junction to fold in a similar manner to $\mathrm{Mg}^{2+}$, but the efficiency is much lower for the monovalent ion. At low concentrations, $\mathrm{Na}^{+}$can screen the interactions between $\mathrm{Mg}^{2+}$ and the junction, leading to a destabilisation of the stacked structures and an acceleration of the conformational transitions.

\section{Discussion}

We have shown that the $4 \mathrm{H}$ junction derived from the hairpin ribozyme interconverts between the parallel and antiparallel forms of both stacking conformers. In a previous study of the hairpin ribozyme and its junction ${ }^{5}$ we were able to identify one of the four states, but the other three could not be resolved. By measuring changes in FRET of the $A B$ and $A D$ vectors we have directly observed transitions to and from the antiparallel forms of both stacking conformers. Changes in FRET of the DB vector have revealed transitions between parallel and antiparallel forms. Although we cannot resolve stacking conformers with this vector, it seems reasonable to assume that both stacking conformers can adopt a parallel conformation. Transitions between states occur within 
the time resolution of our apparatus ( $33 \mathrm{~ms}$ ) with no observable intermediate.

Previous steady-state ensemble studies have found that the binding of a $\mathrm{Mg}^{2+}$ to the junction produces an increase in the FRET of the $\mathrm{AB}$ vector, consistent with the stabilisation of the antiparallel, $\mathrm{D}$ on A conformer. ${ }^{17,22}$ Our studies have shown that there is indeed a stabilisation of this conformer, but that much of the increase in ensemble FRET is due to an increase in the FRET of this conformer with increasing $\mathrm{Mg}^{2+}$. This may be due to a bound $\mathrm{Mg}^{2+}$ stabilising a structure with a reduced distance between the fluorophores in the $\mathrm{AB}$ vector.

In principle, each observable transition between high and low FRET states might result from a rotation between parallel and antiparallel forms without unstacking of the helices, or from passage through an unstacked open intermediate (Figure 9(a)). It is therefore important to understand to what extent rotation and unstacking contribute to the dynamics of the $4 \mathrm{H}$ junction. Although the full kinetic scheme is too complex to solve, we can consider special cases. If $k_{\text {rotation }} \gg$ $k_{\text {unstack }}$ then the kinetic scheme simplifies to that in Figure $9(\mathrm{~b})$. In this case, $k_{\mathrm{b}_{-} \mathrm{DB}}[\mathrm{DB}]=k_{\mathrm{b}_{-} \mathrm{AB}}[\mathrm{AB}]+$ $k_{\mathrm{b}_{\_} \mathrm{AD}}[\mathrm{AD}]$. This is clearly not the case at any $\mathrm{Mg}^{2+}$ concentration (Table 1, Figure 4), so we may conclude that unstacking makes a significant contribution to the observed rates. If $k_{\text {rotation }} \ll$ $k_{\text {unstack }}$ then the kinetic scheme simplifies to that in Figure 9(c). Then it can be shown that $k_{\mathrm{f}_{-} \mathrm{AB}}<$ $k_{\mathrm{b}_{-} \mathrm{AD}}+k_{\mathrm{b}_{-} \mathrm{DB}}$. Equivalent relationships exist for $k_{\mathrm{f}} \mathrm{AD}$ and $k_{\mathrm{f}} \mathrm{DB}$. Each is true at all $\mathrm{Mg}^{2+}$ concentrations. However the reverse relationships $\left(k_{\mathrm{b}_{-} \mathrm{AB}}<k_{\mathrm{f}_{-} \mathrm{AD}}+k_{\mathrm{f}_{-} \mathrm{DB}}\right.$, and the equivalent for $k_{\mathrm{b} \_\mathrm{AD}}$ and $\left.k_{\mathrm{b} \_\mathrm{DB}}\right)$ are not fully satisfied by our data. Thus, this simplified scheme does not account for our data. Either rotation or partial unstacking could contribute to the mechanisms of interconversion. However, as our data more nearly satisfy the conditions for $k_{\text {rotation }} \ll k_{\text {unstack }}$, and given the equivalent activation enthalpy for each vector at $20 \mathrm{mM} \mathrm{Mg} \mathrm{Mg}^{2+}$, it seems likely that unstacking makes the more significant contribution. If so, the observed $k_{\mathrm{b}}$ values will approximate the true rates of unstacking.

The most pronounced effect of $\mathrm{Mg}^{2+}$ on the dynamics of the junction is a significant decrease in the rate of transitions with increasing $\mathrm{Mg}^{2+}$ concentration. Presumably $\mathrm{Mg}^{2+}$ stabilises the stacked conformations making the unstacking of helices less likely, thereby slowing the conformational transitions. All three vectors show similar behaviour in both forward and reverse directions, giving a linear decrease in rate with increasing $\mathrm{Mg}^{2+}$ concentration in a $\log -\log$ plot (hence a power law dependence), except at very high $\mathrm{Mg}^{2+}$ concentrations where the slope is reduced. $\mathrm{Mg}^{2+}$ increases the overall ionic strength, and can also bind at specific sites. We suggest that both mechanisms are responsible for the effects of $\mathrm{Mg}^{2+}$ on transition rates, with the specific binding of $\mathrm{Mg}^{2+}$ (a)

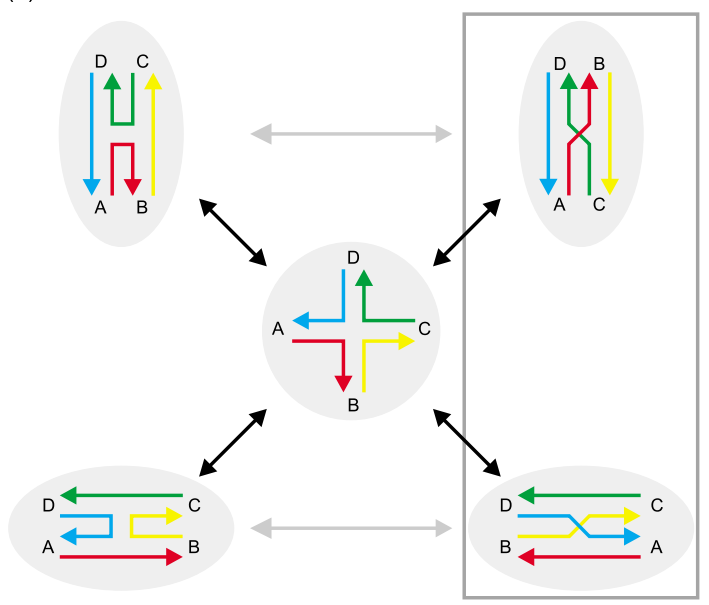

(b)

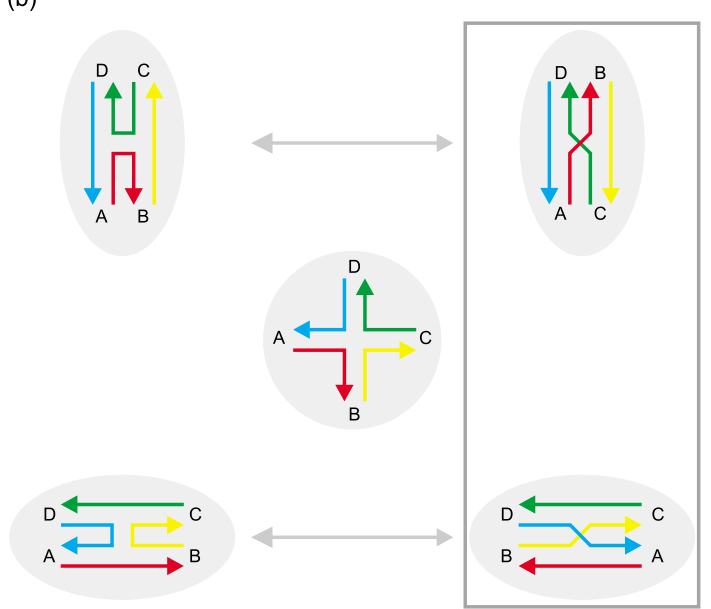

(c)

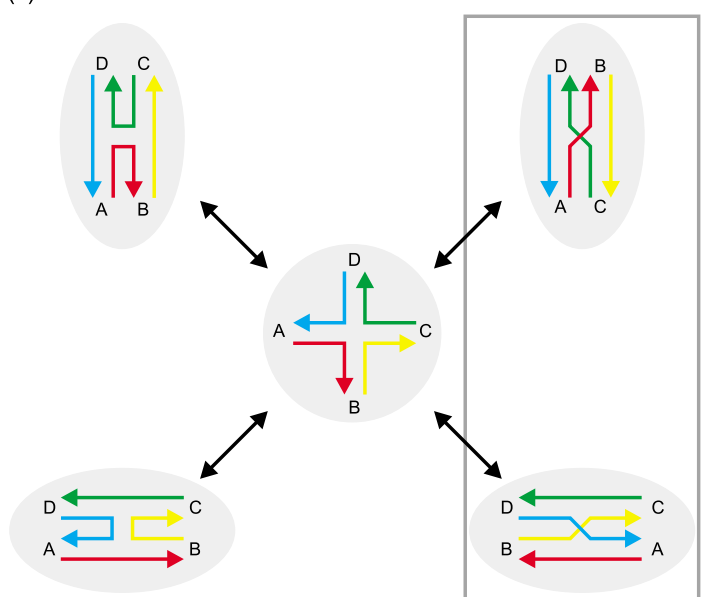

Figure 9. Folding pathways for the hairpin junction. The conformers are as arranged in Figure 1. The two parallel states are boxed to indicate that they cannot be resolved in our experiments. (a) Minimal kinetic scheme for transition between the four stacked conformers, allowing for rotation between parallel and antiparallel states and exchange of stacking conformers via an open intermediate. (b) Reduced kinetic scheme assuming that $k_{\text {rotation }} \gg k_{\text {unstack }}$. (c) Reduced kinetic scheme assuming that $k_{\text {rotation }} \ll k_{\text {unstack }}$. 
(a)
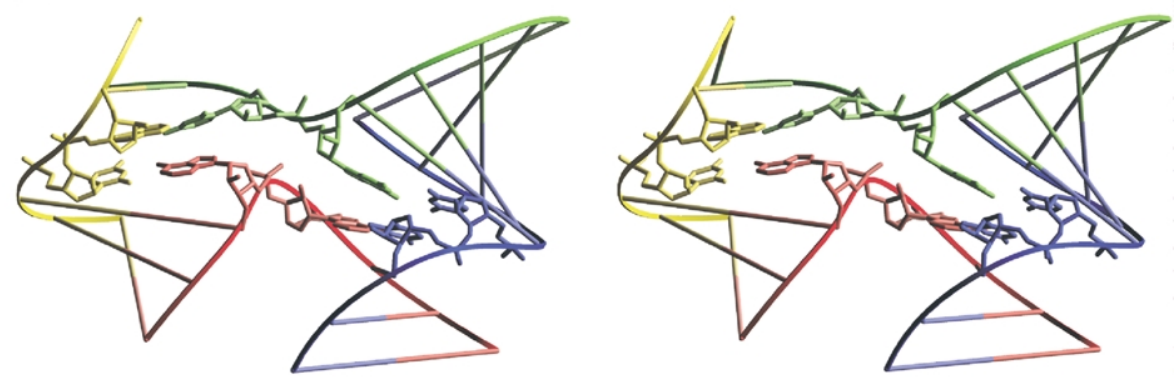

(b)
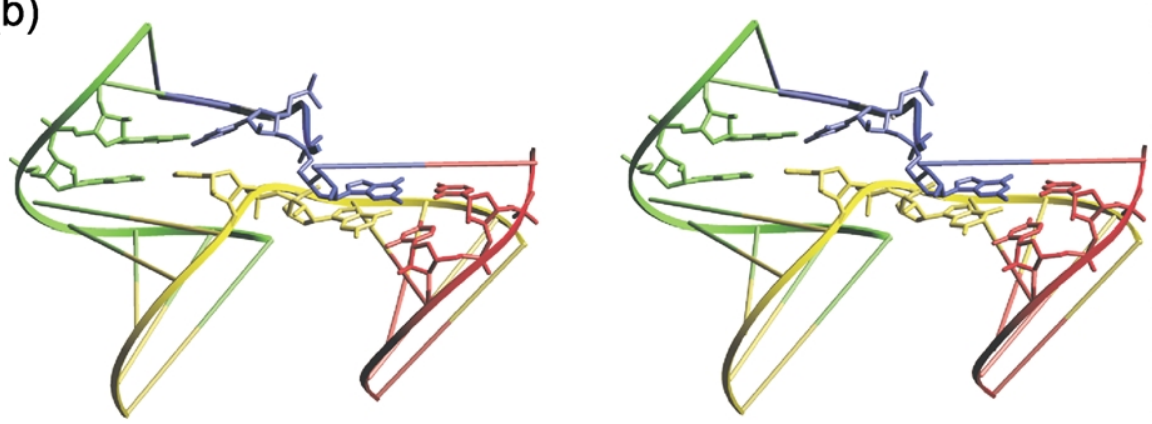

(c)
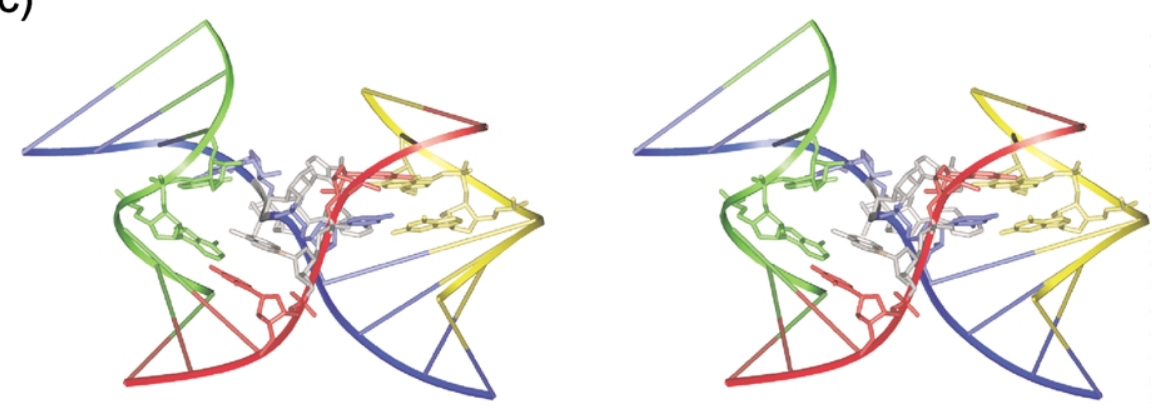

Figure 10. The structures of some representative four-way RNA junctions observed in crystal structures. Parallel-eye stereographic representation of three different four-way junctions derived by crystallography. In each case the trajectories of the backbones are shown by the ribbons with base-pairs indicated by cross-bars, with the four basepairs flanking the point of strand exchange represented in full atom stick manner. (a) The antiparallel structure of the $4 \mathrm{H}$ junction of the hairpin ribozyme as it exists in the full ribozyme, i.e. including the interaction between the loops. ${ }^{25}$ The colouring of the strands matches that used in Figure 1. (b) An antiparallel $4 \mathrm{H}$ junction that exists in the RNaseP RNA. $^{26}$ (c) A parallel $2 \mathrm{HS}_{1} 2 \mathrm{HS}_{2}$ junction formed by helices 94 to 97 of the $23 \mathrm{~S}$ rRNA in the $50 \mathrm{~S}$ ribosomal subunit. ${ }^{30}$ The formally unpaired bases are coloured grey in this representation.

resulting in the change in behaviour at high concentrations. In the presence of monovalent ions the transition rates are higher, and there is a significant population of the unstacked conformation. This indicates that the stacked structure is less energetically favoured in the absence of divalent cations.

Single-molecule FRET analysis shows that the $4 \mathrm{H}$ RNA junction is highly polymorphic and dynamic. The behaviour of the RNA junction bears remarkable similarity to that of the DNA four-way junction in that the rates of conformational transitions are strong functions of cation concentrations, accelerating at lower $\mathrm{Mg}^{2+}$ concentrations. ${ }^{15}$ A major difference, however, exists between the DNA and RNA four-way junctions. While parallel conformations can be stably populated in the RNA case, no evidence for parallel conformations has been observed in similar single-molecule experiments on a DNA junction (C. Joo, S. A. McKinney, D.M.J.L. \& T.H., unpublished observations). This probably results from the stabilisation of the antiparallel structure by the favourable backbone-major groove interaction for the DNA structure, which is not available to an $A$-form helix.

The four-way junction of the hairpin ribozyme samples both parallel and antiparallel structures, 
Table 2. Transition rate constants for the three vectors of the hairpin junction in the presence of $10 \mathrm{mM}$ Hepes $(\mathrm{pH} 7.5), 1 \mathrm{M} \mathrm{NaCl}$ in the absence of $\mathrm{Mg}^{2+}$

\begin{tabular}{ccccc}
\hline & $k_{\mathrm{f}}\left(\mathrm{s}^{-1}\right)$ & $k_{\mathrm{b}}\left(\mathrm{s}^{-1}\right)$ & $P_{\text {dwell time }}(\%)$ & $P_{\text {rate constants }}(\%)$ \\
\hline AB & $2.15 \pm 0.04$ & $1.41 \pm 0.03$ & 63 & 61 \\
DB & $0.99 \pm 0.02$ & $1.21 \pm 0.05$ & 41 & 45 \\
AD & $0.76 \pm 0.02$ & $0.99 \pm 0.05$ & 41 & 44 \\
& & & Sum $=145$ & Sum $=150$ \\
\hline
\end{tabular}

The probabilities of a vector being in the high-FRET state calculated from rate constants and dwell times are also shown.

as well as both stacking conformers. It is likely that all $4 \mathrm{H}$ RNA junctions will exhibit similar conformational flexibility. However, the exact relative populations of the different species are expected to be dependent on local sequence at the branch point. For the hairpin junction the major conformer is the antiparallel structure with D on A stacking, which is the form that juxtaposes the loops of the complete ribozyme. We have shown that it is this presentation of the loops that results in acceleration of the folding of the ribozyme by three orders of magnitude. ${ }^{5}$ Conversely, the interaction between the loops in the fully folded ribozyme locks the junction in the antiparallel form, as seen in the crystal structure of the ribozyme ${ }^{23}$ (Figure 10(a)). Another example of a $4 \mathrm{H}$ junction is found within the specificity domain of the RNA of RNaseP; one arm has a bulged nucleotide after a single basepair, but this does not interact with the junction. In the recent crystal structure ${ }^{24}$ it can be seen that this junction is antiparallel (Figure 10(b)), and quite similar conformationally to the hairpin junction (Figure 10(a)). Thus there appears to be a tendency for $4 \mathrm{H}$ RNA junctions to adopt the antiparallel structure, in agreement with earlier ensemble solution studies (Table 2). ${ }^{14,17,25}$

The significant populations of minor forms observed in the hairpin junction indicates that they do not differ greatly in free energy from the major antiparallel form, and thus could be stabilised by additional factors. These could include the presence of additional unpaired bases close to the junction, by tertiary interactions and by the binding of proteins. We have recently made a study of the $2 \mathrm{HS}_{1} 2 \mathrm{HS}_{2}$ four-way junction (helixhelix-(extra unpaired base)-helix-helix-(two extra unpaired bases) $)^{9}$ of the hepatitis $C$ virus IRES element. ${ }^{26}$ Time-resolved FRET experiments indicate that this junction has approximately equal populations of parallel and antiparallel forms in the presence of divalent metal ions. This junction has been crystallised in an almost side-by-side parallel conformation, ${ }^{27}$ suggesting that the crystallisation process has drawn the equilibrium into the parallel form. Further evidence for flexibility of RNA junctions can be seen by examination of other four-way junctions observed in crystal structures. A number of four-way junctions occur in $23 \mathrm{~S}$ rRNA. Almost none is a $4 \mathrm{H}$ junction, and the great majority adopt a parallel geometry. ${ }^{28}$ For example, a four-way junction that occurs towards the $3^{\prime}$ end of the $23 \mathrm{~S}$ rRNA is (like the IRES junction) a $2 \mathrm{HS}_{1} 2 \mathrm{HS}_{2}$ junction. This adopts a parallel conformation in the $50 \mathrm{~S}$ ribosomal subunit (Figure 10(c)). ${ }^{28}$ The structure appears to be stabilised by unpaired adenine bases on the crossover strand, forming an A-minor interaction in the groove of the junction at the point of strand exchange; the conformation could also be affected by tertiary interactions, and the binding of proteins. Similar A-minor interactions apparently stabilise the parallel forms of the IRES junction ${ }^{29}$ and of another four-way junction (in which one "helix" is a single base-pair) that binds the ribosomal L11 protein, ${ }^{29,30}$ where protein-RNA interactions are also important.

In summary, the analysis of the $4 \mathrm{H}$ junction of the hairpin ribozyme shows a bias towards the antiparallel form of one stacking conformer but other conformations are still significantly populated. Interconversion between different conformations is rapid, occurring at least several times a second under physiologically relevant conditions. This highly polymorphic and dynamic structure may provide an ideal platform for Nature to operate on, that is, either additional structural elements within the RNA or protein cofactors may selectively stabilise a particular conformation desired for biological function.

\section{Materials and Methods}

\section{Preparation of RNA junctions}

RNA was synthesised using $t$-BDMS chemistry, except for $\mathrm{Cy}-5$ labelled species, which were purchased from Dharmacon. The $4 \mathrm{H}$ junction derived from the hairpin ribozyme was assembled from oligonucleotides of the following sequences (all written $5^{\prime}$ to $3^{\prime}$ ):

a strand: cCGACAGGACUGUCAACCAGGUAAU

AUACCACUUGCGg (unlabelled and 5'-Cy3)

b strand: cCGCAAGUGGUAUAUUACCUGGUA CGCGUUCACGg (unlabelled and 5'-Cy5)

c strand: cCGUGAACGCGUGGUGCGAAUCGg (5'-biotin)

d strand: cCGAUUCGCACCUGACaGUCCUGUC $\mathrm{Gg}$ (unlabelled, 5'-Cy3 and 5'-Cy5)

Lowercase letters denote 2'-deoxyribonucleotides. The appropriate four strands required to generate a particular vector were annealed by heating the strands to $70{ }^{\circ} \mathrm{C}$ in $10 \mathrm{mM}$ Hepes ( $\mathrm{pH} 7.5), 50 \mathrm{mM} \mathrm{NaCl}$, followed by slow cooling to room temperature. To ensure that all of the Cy3-labelled strand is incorporated into complexes comprising four strands, the concentrations of the other three strands were in $20-50 \%$ excess. Non-denaturing gel electrophoresis has shown that the concentrationlimiting strand is fully incorporated into four-strand complexes under these conditions.

\section{Single molecule experiments}

A narrow channel was made between a cleaned quartz microscope slide (Finkenbeiner) and a coverslip using double-sided adhesive tape. RNA was attached to 
the surface by successive additions of $40 \mu \mathrm{l}$ of $1 \mathrm{mg} / \mathrm{ml}$ biotinylated BSA (Sigma), $40 \mu \mathrm{l}$ of $0.2 \mathrm{mg} / \mathrm{ml}$ streptavidin (Molecular Probes) and $40 \mu \mathrm{l}$ of $10-50 \mathrm{pM}$ biotinylated RNA in $10 \mathrm{mM}$ Tris- $\mathrm{HCl}(\mathrm{pH} 8.0), 50 \mathrm{mM} \mathrm{NaCl}$ (TN buffer). Each addition was incubated for ten minutes, and followed by washing with TN buffer. The concentration of the RNA solution was adjusted to give good surface density for single-molecule experiments. After checking that fluorescent spots were well separated from one another, we injected $60 \mu \mathrm{l}$ of $10 \mathrm{mM}$ Hepes ( $\mathrm{pH}$ 7.5) with salt concentrations given in the text, and an oxygen scavenging system composed of $6 \%$ $(\mathrm{w} / \mathrm{v})$ glucose, $1 \%$ 2-mercaptoethanol, $0.1 \mathrm{mg} / \mathrm{ml}$ glucose oxidase (Sigma) and $0.02 \mathrm{mg} / \mathrm{ml}$ catalase (Sigma). The surface density of RNA molecules did not decrease significantly over several hours, suggesting that nuclease contamination is minimal. The wide-field microscope is based on an inverted microscope (Olympus IX70) with a $60 \times$ water immersion objective with numerical aperture of 1.2 (Olympus, Melville, NY) and an intensified CCD camera (Intensified Pentamax, Roper Scientific, Trenton, $\mathrm{NJ})^{31}$ that can record both the donor and acceptor intensities from several hundred single molecules simultaneously. Solid-state $532 \mathrm{~nm}$ lasers (Crystalaser) were used to excite the molecules via an evanescent field generated using a prism. Data were acquired using software written in Visual C ++ (Microsoft). The time resolution of data acquisition was either $100 \mathrm{~ms}$ or $33 \mathrm{~ms}$, depending on the observed rate of conformational transitions. Two time bins gave identical rates for slow conformational transitions. The temperature of the sample was regulated with a water-circulating bath (Neslab) flowing to copper tubing in contact with (i) a home-built brass collar on the objective, (ii) the plate that holds the sample cell, and (iii) metal pieces holding the prism. The temperature was measured with a thermocouple sandwiched between a quartz slide and coverslip and placed on the microscope in place of the sample. The error in temperature measurement is estimated to be less than 1 deg. C.

\section{Determination of rates by dwell time analysis and auto-correlation}

After correcting for donor leakage into the acceptor channel, the apparent FRET efficiency $E_{\text {app }}$ was calculated using $E_{\mathrm{app}}=I_{\mathrm{A}} /\left(I_{\mathrm{A}}+I_{\mathrm{D}}\right)$, where $I_{\mathrm{A}}$ and $I_{\mathrm{D}}$ are the intensities of the sensitised emission of the acceptor and the donor emission, respectively. Histograms of dwell times of high and low-FRET states were generated using data acquired from approximately 200 molecules. These were fitted by an exponential decay function to obtain rate constants $\left(k_{\mathrm{f}}\right.$ and $\left.k_{\mathrm{b}}\right)$. For temperature dependence studies, auto-correlation analysis was used to obtain the rate constants. An $E_{\mathrm{app}}$ time trace, $E_{\mathrm{app}}(t)$, was made by combining data from more than 40 molecules under each condition. The auto-correlation function was calculated according to $\int E_{\mathrm{app}}(t) E_{\mathrm{app}}(t-\Delta t) \mathrm{d} t$. For a two-state fluctuation, the auto-correlation function $A C(\Delta t)$ takes the form $A \mathrm{e}^{-\Delta t / \tau}$ where $\tau$ is the correlation time and is an inverse of the sum of the forward and backward rates.

\section{Acknowledgements}

We are grateful for financial support from the
National Institutes of Health, the National Science Foundation, and the Searle scholars foundation (T.H.) and Cancer Research-UK (D.M.J.L.).

\section{References}

1. Murchie, A. I. H., Thomson, J. B., Walter, F. \& Lilley, D. M. J. (1998). Folding of the hairpin ribozyme in its natural conformation achieves close physical proximity of the loops. Mol. Cell, 1, 873-881.

2. Walter, N. G., Burke, J. M. \& Millar, D. P. (1999). Stability of hairpin ribozyme tertiary structure is governed by the interdomain junction. Nature Struct. Biol. 6, 544-549.

3. Fedor, M. J. (1999). Tertiary structure stabilization promotes hairpin ribozyme ligation. Biochemistry, 38, 11040-11050.

4. Zhao, Z. Y., Wilson, T. J., Maxwell, K. \& Lilley, D. M. J. (2000). The folding of the hairpin ribozyme: dependence on the loops and the junction. $R N A, \mathbf{6}$, 1833-1846.

5. Tan, E., Wilson, T. J., Nahas, M. K., Clegg, R. M., Lilley, D. M. J. \& Ha, T. (2003). A four way junction accelerates hairpin ribozyme folding via a discrete intermediate. Proc. Natl Acad. Sci. USA, 100, 9308.

6. Pley, H. W., Flaherty, K. M. \& McKay, D. B. (1994). Three-dimensional structure of a hammerhead ribozyme. Nature, 372, 68-74.

7. Scott, W. G., Finch, J. T. \& Klug, A. (1995). The crystal structure of an all-RNA hammerhead ribozyme: a proposed mechanism for RNA catalytic cleavage. Cell, 81, 991-1002.

8. Lafontaine, D. A., Norman, D. G. \& Lilley, D. M. (2002). The global structure of the VS ribozyme. $E M B O$ J. 21, 2461-2471.

9. Lilley, D. M. J., Clegg, R. M., Diekmann, S., Seeman, N. C., von Kitzing, E. \& Hagerman, P. (1996). A nomenclature of junctions and branchpoints in nucleic acids-recommendations 1994. J. Mol. Biol. 255, 554-555.

10. Holliday, R. (1964). A mechanism for gene conversion in fungi. Genet. Res. 5, 282-304.

11. Lilley, D. M. J. (2000). Structure of helical junctions in nucleic acids. Q. Rev. Biophys. 33, 109-159.

12. Duckett, D. R., Murchie, A. I., Diekmann, S., von Kitzing, E., Kemper, B. \& Lilley, D. M. (1988). The structure of the Holliday junction, and its resolution. Cell, 55, 79-89.

13. Murchie, A. I., Clegg, R. M., von Kitzing, E., Duckett, D. R., Diekmann, S. \& Lilley, D. M. (1989). Fluorescence energy transfer shows that the four-way DNA junction is a right-handed cross of antiparallel molecules. Nature, 341, 763-766.

14. Duckett, D. R., Murchie, A. I. H. \& Lilley, D. M. J. (1995). The global folding of four-way helical junctions in RNA, including that in U1 SnRNA. Cell, 83, 1027-1036.

15. McKinney, S. A., Declais, A. C., Lilley, D. M. J. \& Ha, T. (2003). Structural dynamics of individual Holliday junctions. Nature Struct. Biol. 10, 93-97.

16. Berzal-Herranz, A., Joseph, S., Chowrira, B. M., Butcher, S. E. \& Burke, J. M. (1993). Essential nucleotide sequences and secondary structure elements of the hairpin ribozyme. EMBO J. 12, 2567-2573.

17. Walter, F., Murchie, A. I. H. \& Lilley, D. M. J. (1998). Folding of the four-way RNA junction of the hairpin ribozyme. Biochemistry, 37, 17629-17636.

18. Ha, T., Enderle, T., Ogletree, D. F., Chemla, D. S., 
Selvin, P. R. \& Weiss, S. (1996). Probing the interaction between two single molecules-fluorescence resonance energy transfer between a single donor and a single acceptor. Proc. Natl Acad. Sci. USA, 93, $6264-6268$.

19. Ha, T., Zhuang, X. W., Kim, H. D., Orr, J. W., Williamson, J. R. \& Chu, S. (1999). Ligand-induced conformational changes observed in single RNA molecules. Proc. Natl Acad. Sci. USA, 96, 9077-9082.

20. Zhuang, X. W., Bartley, L. E., Babcock, H. P., Russell, R., Ha, T. J., Herschlag, D. \& Chu, S. (2000). A single-molecule study of RNA catalysis and folding. Science, 288, 2048-2051.

21. Zhuang, X. W., Kim, H., Pereira, M. J. B., Babcock, H. P., Walter, N. G. \& Chu, S. (2002). Correlating structural dynamics and function in single ribozyme molecules. Science, 296, 1473-1476.

22. Wilson, T. J. \& Lilley, D. M. (2002). Metal ion binding and the folding of the hairpin ribozyme. RNA, 8, 587-600.

23. Rupert, P. B. \& Ferre-D'Amare, A. R. (2001). Crystal structure of a hairpin ribozyme-inhibitor complex with implications for catalysis. Nature, 410, 780-786.

24. Krasilnikov, A. S., Yang, X., Pan, T. \& Mondragon, A. (2003). Crystal structure of the specificity domain of ribonuclease P. Nature, 421, 760-764.

25. Walter, F., Murchie, A. I. H., Duckett, D. R. \& Lilley,
D. M. J. (1998). Global structure of four-way RNA junctions studied using fluorescence resonance energy transfer. RNA, 4, 719-728.

26. Melcher, S. E., Wilson, T. J. \& Lilley, D. M. (2003). The dynamic nature of the four-way junction of the hepatitis $\mathrm{C}$ virus IRES. RNA, 9, 809-820.

27. Kieft, J. S., Zhou, K., Grech, A., Jubin, R. \& Doudna, J. A. (2002). Crystal structure of an RNA tertiary domain essential to HCV IRES-mediated translation initiation. Nature Struct. Biol. 9, 370-374.

28. Ban, N., Nissen, P., Hansen, J., Moore, P. B. \& Steitz, T. A. (2000). The complete atomic structure of the large ribosomal subunit at $2.4 \AA$ resolution. Science, 289, 905-920.

29. Conn, G. L., Draper, D. E., Lattman, E. E. \& Gittis, A. G. (1999). Crystal structure of a conserved ribosomal protein-RNA complex. Science, 284, 1171-1174.

30. Wimberly, B. T., Guymon, R., McCutcheon, J. P., White, S. W. \& Ramakrishnan, V. (1999). A detailed view of a ribosomal active site: the structure of the L11-RNA complex. Cell, 97, 491-502.

31. Ha, T., Rasnik, I., Cheng, W., Babcock, H. P., Gauss, G., Lohman, T. M. \& Chu, S. (2002). Initiation and reinitiation of DNA unwinding by the Escherichia coli Rep helicase. Nature, 419, 638-641.

Edited by J. Doudna

(Received 30 September 2003; received in revised form 20 November 2003; accepted 2 December 2003) 